\title{
Approaches to Automatic Seabed Classification
}

\author{
Enrique Coiras, David Williams \\ NATO Undersea Research Centre \\ 19126 La Spezia. Italy
}

\section{Introduction}

The latest advances in sonar technology permit to observe the underwater environment with improved resolution and coverage, with the latest side-scan and synthetic aperture sonar (SAS) systems able to produce images with a resolution of a few centimetres. This capability enables the seabed configuration to be determined relatively easily by visual inspection of the sonar images - rocks, sand ripples and underwater plants can be clearly identified visually, for instance. This process of seafloor characterization can be automated by analyzing the characteristics of the image texture at any given point. The resulting classification is useful for diverse applications, including environmental monitoring, security, and defence.

Seabed classification is the process by which one divides (or segments) an image of a typically large area of seabed into different regions based on their local characteristics. The characteristics (or features) used for classification and the specific classes chosen will depend on the application.

For mine countermeasures (MCM), for instance, an area may be segmented into flat, rippled and complex regions, which roughly divide the surveyed area into sub-regions of increasing mine-hunting difficulty. Another example could be the estimation of fishing density in an area over time, which can be determined by examining the number of trawl marks on the seabed.

Regardless of the particular application, the basic desired output of a seabed classification algorithm is a 'map' of the seabed indicating where each seabed type is present. Furthermore, as with more traditional remote sensing modalities, analyzing collected data in an automated manner is vital when dealing with vast quantities of data.

In this chapter, approaches to automatic classification of seabed into different types or classes are discussed. The chapter is organized as follows. Section 2 gives an overview of the different general approaches for seabed classification. Section 3 discusses more detailed aspects of these general approaches, such as feature extraction, classifiers, and fusion of multiple views. In Section 4, a few specific classification approaches, which span the variety of possible methods, are described. Example results on real sonar imagery are also shown for these particular approaches. Finally, conclusions are summarized in Section 5. 


\section{Overview}

Many different approaches for addressing the task of automatic seabed classification exist. One useful categorization of these approaches is based on the knowledge that is possessed about the problem. From this point of view, all seabed classification approaches can be divided into three broad categories.

In the most ideal case, a priori knowledge about specific seabed types that one will encounter is available. This information in turn permits a model-based approach, in which a mathematical model that characterizes certain specific seabed types can be constructed. One can then use the degree to which a portion of seabed matches the defined model as a metric for performing classification. The main advantage of a model-based approach is that the algorithm can be tailored specially for a seabed type of particular interest.

Unfortunately, it is usually difficult or impossible to accurately model most types of seabed. Because of the characteristic complexity or natural variations within a given type of seabed, one must therefore typically employ an alternative seabed classification approach that assumes less specific knowledge of the problem. Another disadvantage of model-based approaches is their poor flexibility, being very specific and requiring new models for any new classes that may have to be added to the system.

Rather than knowing detailed information about certain seabed types to be encountered, one may instead know only about general classes of seabed types. Often, this general knowledge is provided in the form of labelled training data. That is, data for which the true identity of different seabed types from some location is possessed. When such information is available and is exploited, the resulting approach is referred to as supervised.

The advantage of employing a supervised approach is that the resulting classification of the seabed will be in terms of types or classes known a priori. Thus, the resulting segmentation of the seabed will be in terms of specific seabed types that are important for the application at hand. As briefly mentioned in the introduction, MCM operations typically seek to segment an area of seabed into one of three basic types -- flat, benign seabed; seabed characterized by sand ripples; and complex seabed that contains rocks or other clutter objects. By possessing labelled training data (or ground-truth) of these three classes, an algorithm that seeks to discriminate among these seabed types can be developed.

One drawback to the use of supervised approaches for seabed classification is that the process of acquiring labelled seabed data is expensive and time-consuming. In addition to acquiring the data at sea, a human would be required to tediously hand-label the data as specific seabed types. Moreover, this ground-truthing process is inherently subjective and could vary from one human operator to another.

Another potential source of concern regarding supervised methods is the implicit assumption that the underlying statistics that generated the training and test data are the same. In real applications, this assumption is often violated, leading to what is known as covariate shift (Sugiyama et al., 2007), sample selection bias (Zadrozny, 2004), or concept drift (Widmer \& Kubat, 1996; Liao et al., 2005). For example, if one collects training data and learns a classifier from one site, but then attempts to classify test data collected at a different location, a fundamental mismatch in class statistics can lead to poor classification performance.

This scenario motivates the use of the third general approach to seabed classification, in which no labelled training data is required. This unsupervised approach is appropriate 
when no labelled training data exists or when little a priori information about the seabed types to be encountered is available.

Since in principle any information known in advance about the seabed classification task at hand should be exploited, the relevance of unsupervised classification algorithms arises when no such knowledge is possessed. This lack of knowledge about the problem is reflected by the numerous drawbacks that plague unsupervised approaches. For one, the resulting groups into which the seabed is segmented may not be valuable divisions for the application. Moreover, the number of seabed types must often be specified, though it is not always known a priori. Lastly, with an unsupervised approach, human intervention is required to associate the groups into which the data is segmented with distinct seabed types. Despite these drawbacks, when no information or training data is available, there is little alternative to unsupervised methods.

\section{Features, Classifiers, Outputs, and Fusion}

Model-based seabed classification approaches are specially tailored for specific seabed types and certain scenarios. More commonly, seabed classification approaches fall under the purview of supervised or unsupervised methods. In both supervised and unsupervised approaches, features must be extracted from the seabed and a subsequent classifier must be built. The number of possible combinations of choices for these two requirements is endless. Thus, rather than attempting to provide an exhaustive list of such approaches, a representative sample of commonly used techniques will be presented in this chapter.

The purpose of the feature extraction stage is to represent an image of a given area of seabed in a succinct manner. The features that are extracted should be such that they are capable of discriminating among the different seabed types of interest. That is, the feature values of one particular seabed type should be differentiable from those of other seabed types.

A few examples of types of features useful for seabed classification include moment-based features, wavelet-based features, and features derived from eigendecompositions. Momentbased features calculate certain properties of the distributions of the gray-level pixel intensities for a given area of seabed. These features are motivated by the fundamental sonar scattering physics of the seabed. For example, the amount of acoustic energy scattered back to the sonar receiver from areas of seabed characterized by sand ripples or rocks is larger than the amount scattered from flat, benign seabed.

Features based on wavelet decomposition are popular because they can be used to characterize textural properties of seabed images. Namely, the wavelet coefficient energy will be large when the orientation and scale match the orientation and scale of high-energy texture components in an image (Mallat, 1999).

Feature sets based on spectral clustering (Meila \& Shi, 2000; Ng et al., 2001), which exploits the eigenvectors of a matrix composed of distances between data points, have also been used with success. Spectral clustering will effectively transform a feature vector into a new feature vector in a lower dimensional space by retaining only the eigenvectors corresponding to the largest eigenvalues.

After feature extraction, classification must be performed on the resulting set of feature vectors. The objective of the classification stage is to develop a rule that will successfully discriminate among the various seabed types by discriminating their corresponding feature vectors in feature space. When an unsupervised approach is employed, no training data is 
available to perform the classification and the classification stage typically only clusters the data. Common unsupervised approaches include $k$-means clustering and methods based on modelling the data as mixtures of Gaussians.

When a supervised approach is employed, labelled training data is used to build a classifier to which unlabeled test data is subsequently submitted. Many different discriminative classification approaches exist, such as decision trees (Breiman, 1993), support vector machines (Shawe-Taylor \& Cristianini, 2000), relevance vector machines (Tipping, 2001), and Gaussian processes (Rasmussen \& Williams, 2006).

Depending on the classification approach employed, the result of the algorithm can be in different forms. For example, each area of seabed may be assigned a probability or score of belonging to each seabed type. Alternatively, each area of seabed may be classified as a certain seabed type directly via a hard decision.

When multiple views of an area of seabed are available, different data fusion approaches can be used to combine them. There exist two general approaches to perform such fusion. In one approach, each view of the seabed is considered independently and then the multiple decisions or seabed type scores are combined in some particular manner. In the second approach, all views of the seabed are considered jointly so that a single decision regarding seabed type is produced. An example of the former fusion approach is Dempster-Shafer theory (Shafer, 1976), while an example of the latter fusion approach would be the result of a fully Bayesian framework (Berger, 1993).

\section{Example Classification Approaches and Results}

To provide more specific examples of seabed classification methods, three particular algorithms are described in greater detail in this section. Two of them (model-based and unsupervised) have been applied to SAS images acquired by the MUSCLE autonomous underwater vehicle (AUV), while the other method (supervised) is demonstrated on sidescan data collected by a Remus AUV.

MUSCLE images were obtained by the NATO Undersea Research Centre (NURC) during the Colossus II sea trial in the Baltic Sea off the coast of Latvia. In that trial, high-resolution sonar data was collected by the MUSCLE AUV, which is equipped with a $300 \mathrm{kHz}$ sonar capable of constant image resolution of approximately $3 \mathrm{~cm} \times 3 \mathrm{~cm}$ at ranges up to $200 \mathrm{~m}$. The Remus images were collected during a NURC exercise in Capo Teulada, Sardinia, where an area of five square kilometres was observed by multiple sensors. The Remus vehicle is equipped with a $900 \mathrm{kHz}$ Marine Sonic sonar capable of forming images of about $8.6 \mathrm{~cm} \times 12 \mathrm{~cm}$ in resolution at ranges up to $30 \mathrm{~m}$.

\subsection{Model-based Classification Example}

Model-based classification algorithms are useful when the classification target can be described mathematically. The case of sand ripples exemplifies this situation, given the similarity between sand ripples and a striped pattern or sinusoidal function. By applying image correlation (Lewis, 1995) the response of the image to a bank of predefined filters based on those patterns can be used to quantify the "ripplicity" of every image pixel.

Since a single sinusoidal crest will correlate also with lines or any proud object on the seafloor (showing a highlight followed by a shadow, just like the ripples), the filters are designed to contain three sinusoidal periods - the idea being that only rippled areas will 
respond to them fully. The filters cover a range of orientations and scales so that rippled areas of various sizes and directions can be detected. Figure 1 shows the configuration of the filter bank using six orientations. The angle and size of the individual filter that produces the highest response will give the approximate orientation and size of the ripples in that area. The estimated angles and sizes, however, will only be meaningful when ripples are actually present in the region.
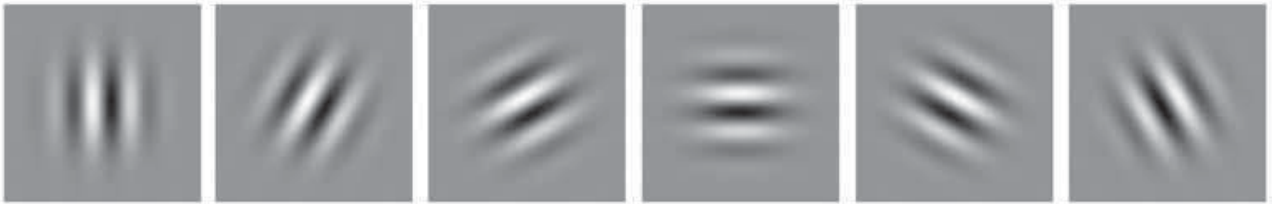

Fig. 1. Filter bank containing six orientations that is used by the model-based ripple classification algorithm.

In order to determine whether a given response value is to be considered high enough to indicate the presence of ripples, a calibration step is used to derive lower and upper thresholds for the responses to the filter bank. In this way the values returned when convolving with the filters can be scaled from 0 to 1 , providing a continuous measure that ranges from "no ripple" to "perfect ripple". The thresholds are obtained by filtering two predetermined images: one containing a synthetic image of alternate black and white bands (which will determine the high threshold for the filters) and an image containing random pixels (which will determine the lower threshold). Both images are tuned to the scale of the filters - the bands being exactly half a period of the sinusoidal component, and the random image being median-filtered to produce blobs of the relevant scale. To ensure the lower threshold is safely set, it is actually obtained as the median response from twenty of those random images.

The specificity of this filter-based method can be further increased by targeting the differences in response to orthogonal directions. The underlying idea being that a rippled area will produce a high response to a ripple filter of the adequate orientation and a low response to the filter tuned to the orthogonal direction, whereas an area showing no ripples will produce responses of similar strength to any filter orientation. This is the approach used to produce the results shown in Figure 2, where ripples have been detected in a MUSCLE SAS image using six orientations and three filter sizes of $0.5,0.6$ and 0.7 meters.

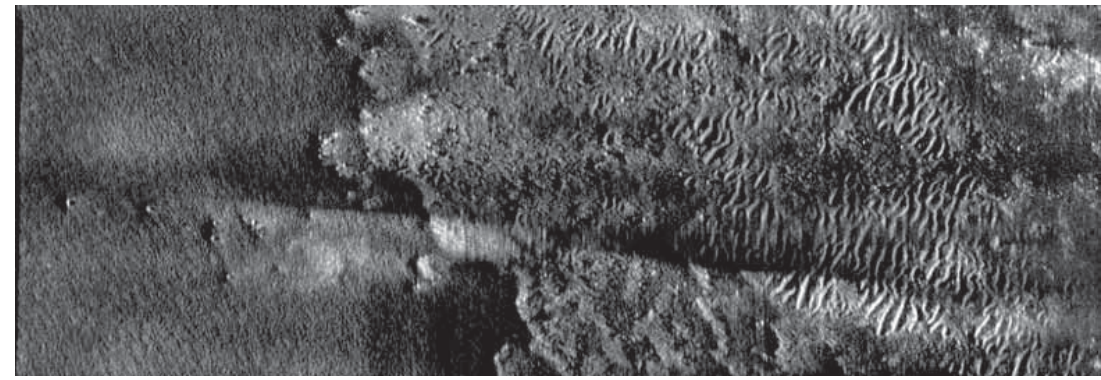




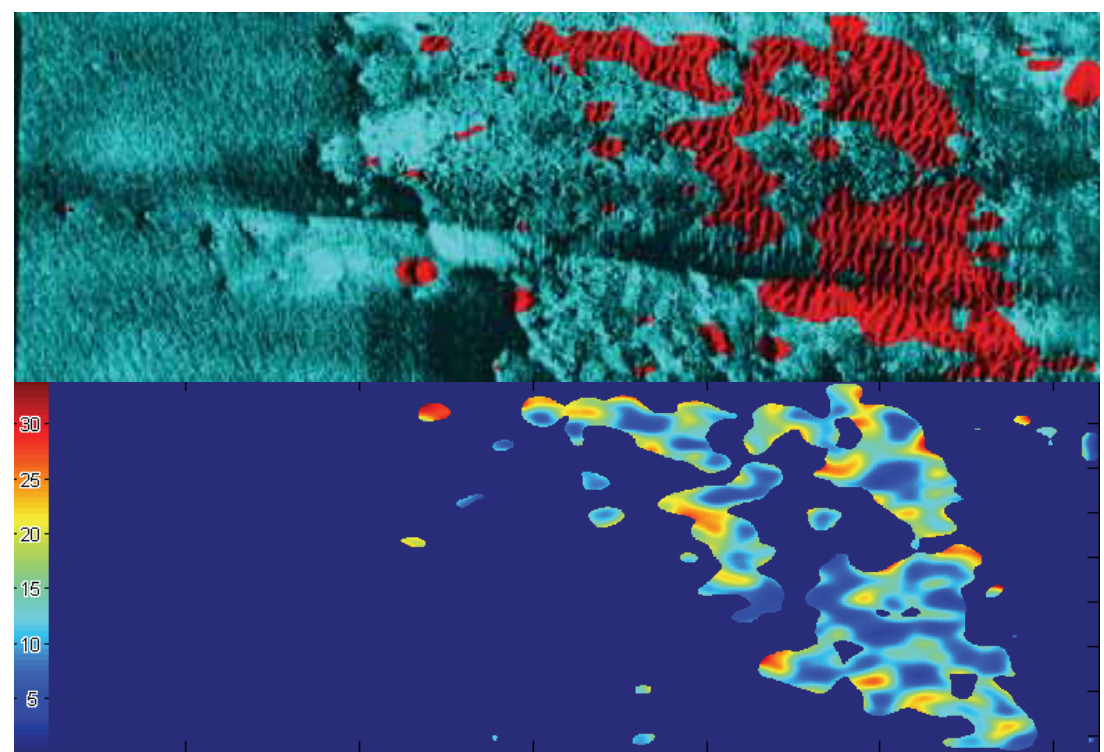

Fig. 2. Results of model-based sand ripple characterization. (a) A MUSCLE SAS seabed image. (b) Segmentation into rippled (red) and non-rippled (cyan) areas. (c) Local ripple orientation in degrees.

\subsection{Supervised Classification Example}

When model-based classification is not feasible or practical, a more general approach is to use a supervised system. In a supervised approach, an algorithm is trained to recognize particular characteristics that permit the assignment of objects to different classes.

A supervised system is more flexible in the sense that there is no need to devise a new model every time a previously unseen class has to be considered. For seabed classification this is especially important when using data from different sensors (Coiras, 2007). The multi-sensor seabed classification system presented here uses an independent supervised binary classifier (detector) for every class considered. The detectors are trained using a given set of ground-truth samples in sensor space (that is, not yet geo-referenced), and their classification performances are estimated in order to determine their individual confusion matrices.

The training samples are manually ground-truthed with an image editing application (in this case, Photoshop). A small set of images from the mission that are representative of the classes to consider are selected, and a binary map is created for each of them. In the training example shown in Figure 3, three binary segmentations have been manually produced for flat, posidonia and rock classes. 


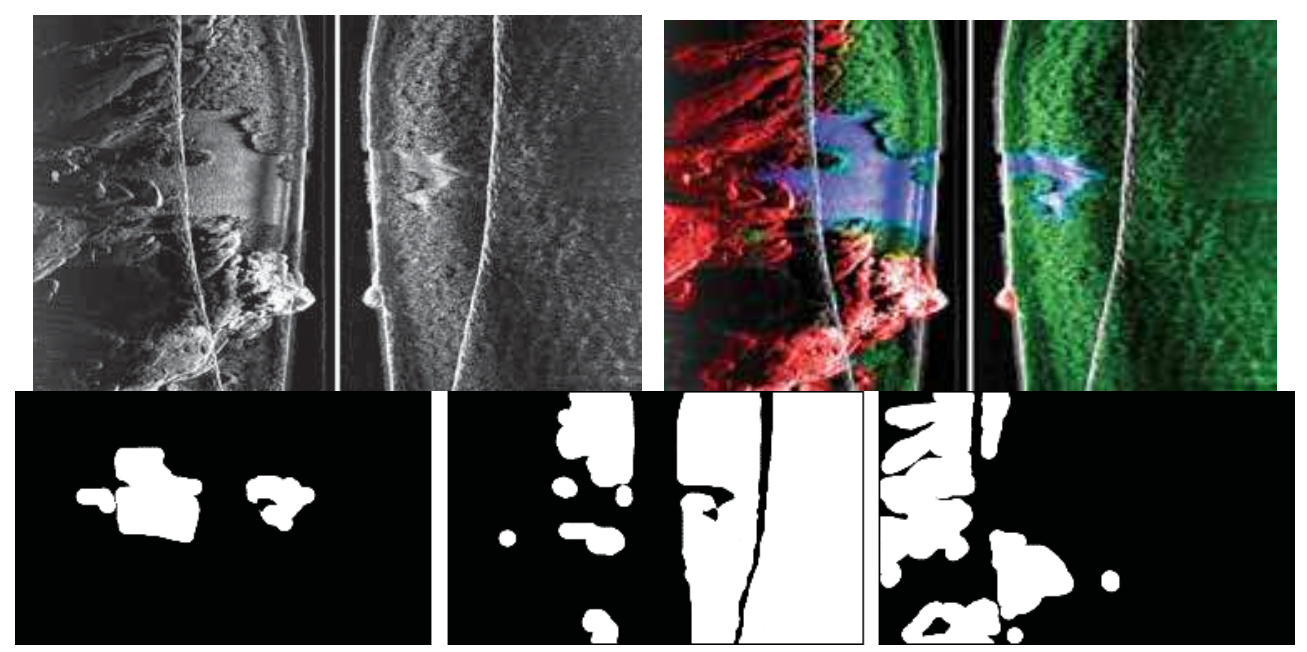

Fig. 3. Manual segmentation of a side-scan image to train a supervised classifier. Counterclockwise from top-left: (a) side-scan image; (b) segments for flat, posidonia and rock areas; (c) colored side-scan image using the flat, posidonia, and rock segment images as blue, green, and red channels.

The subsequent classification is based on texture analysis (Chang \& Kuo, 1993) and uses wavelet decomposition to generate the feature vectors. Four bi-orthogonal wavelets (Mallat, 1999) in two scales generate feature vectors of 16 components that are then classified using a decision tree. This results in three maps for each class, as shown in Figure 4, which correspond to the probability of each image pixel belonging to each of the classes considered. 


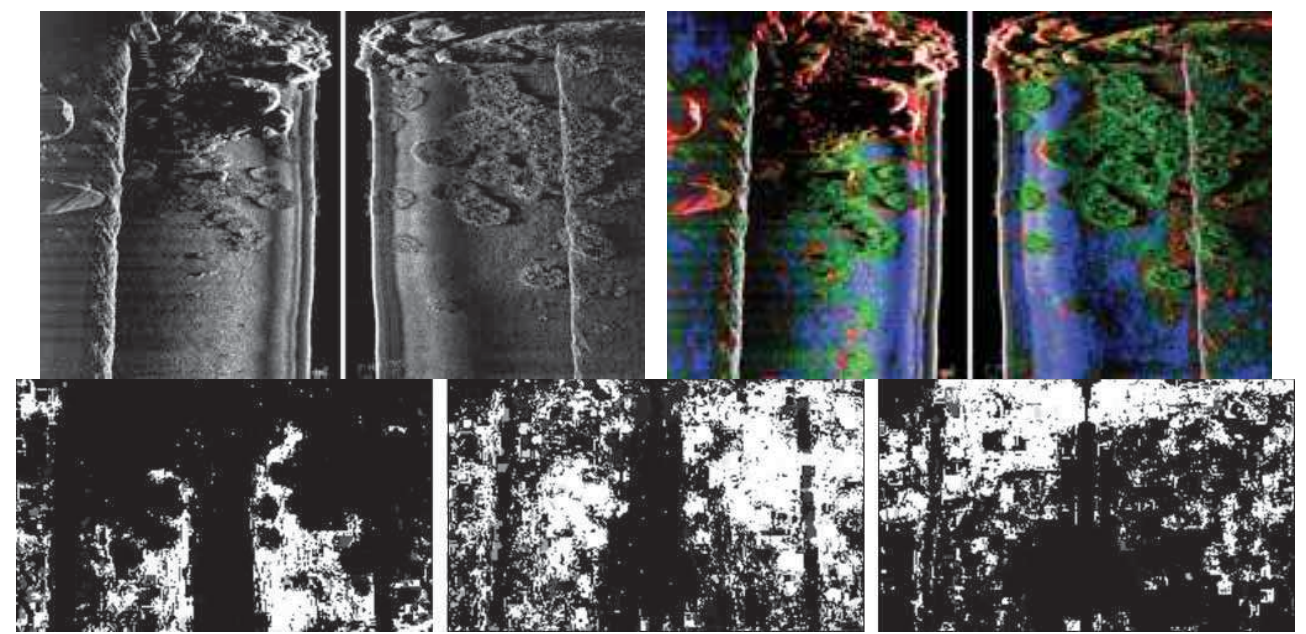

Fig. 4. Supervised segmentation of a side-scan image. Counter-clockwise from top-left: (a) side-scan image; (b) probability images for flat, posidonia and rock classes; (c) colored sidescan image using the flat, posidonia, and rock segment images as blue, green, and red channels.

A decision regarding the actual class of a pixel can be taken at this point by selecting, for instance, the class that has the highest probability. In our case, the decision is delayed until after geo-referencing. This choice is made because in our seabed observation missions, each seafloor point is observed more than once, and ideally all gathered evidence should be used to make a more informed classification decision.

At this stage, the performance of the sensor for each class can be taken into account and used to modulate the probabilities given by the decision tree, as described in (Coiras, 2007) for the multi-sensor case.

After all images have been processed in sensor space, they are geo-referenced into an area mosaic. The different observations available for every seabed point are combined using data fusion. In our case, the Dempster-Shafer theory of evidence (Shafer, 1976) is used because of its ability to cope with conflicting information, which is particularly important for the multisensor case. The result of the data fusion stage is a set of three mosaics of the area composed of the belief that each pixel corresponds to the flat, posidonia, and rock classes.

The final classification map for the observed area is determined by the maximum-belief decision rule, which selects a single class for each of the image pixels. Figure 5 shows the final classification result for the Capo Teulada survey, in south-eastern Sardinia. 


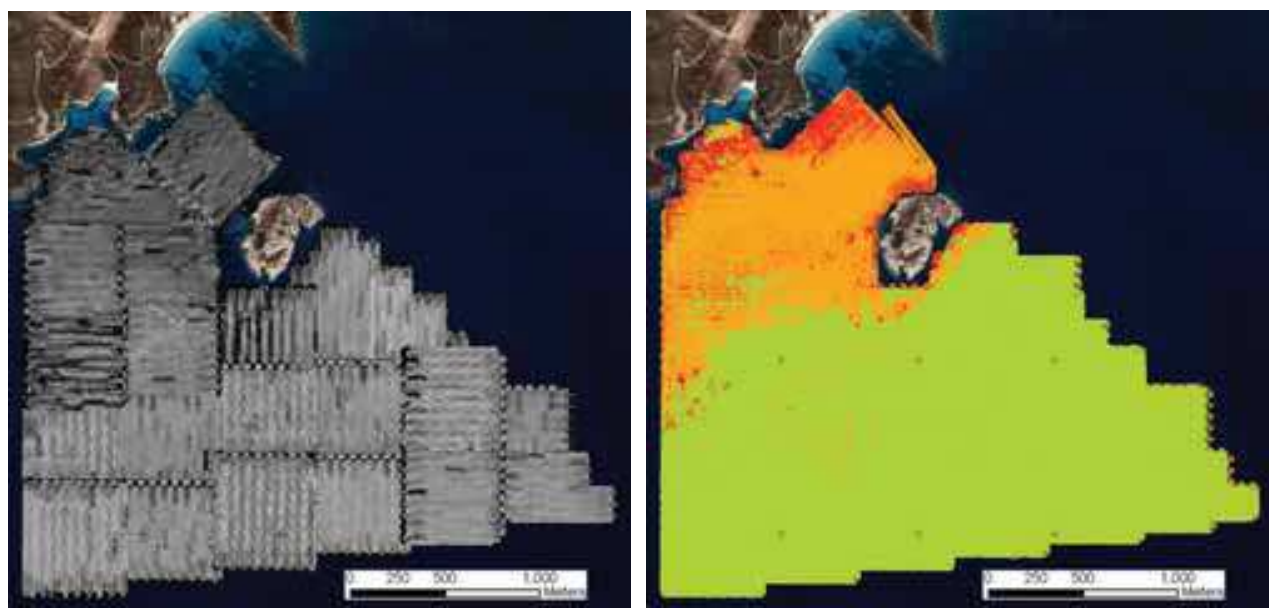

Fig. 5. Results of supervised segmentation for a full side-scan mission covering an area of five square kilometres. (a) Overview of the covered area showing all side-scan tracks. (b) Result of the supervised classification of the seabed into the following three classes: flat seabed (green), posidonia formations (orange) and rocky seabed (red).

\subsection{Unsupervised Classification Example}

Next we consider an unsupervised seabed classification algorithm (or more appropriately, seabed segmentation algorithm, since the seabed classes are not known). In this particular approach, the "atomic" unit for seabed classification is assumed to be a $2 \mathrm{~m} \times 2 \mathrm{~m}$ area of seabed. That is, each $2 \mathrm{~m} \times 2 \mathrm{~m}$ area of seabed corresponds to one data point. This particular size was chosen as a compromise among several factors. The larger the area chosen, the more likely that a single data point will have the unfavourable property of containing multiple types of seabed. However, if the area is too small, the distinguishing characteristics of the seabed that indicate a certain seabed type may be lost.

We consider four different unsupervised segmentation approaches, which differ both in the set of features employed and in the clustering method used. Specifically, segmentation is performed when using (i) moment features with $k$-means clustering, (ii) moment features with spectral clustering, (iii) wavelet features with $k$-means clustering, and (iv) wavelet features with spectral clustering.

The wavelet-based features consist of 16 features that are derived from the coefficients of a bi-orthogonal wavelet decomposition (Mallat, 1999) of each SAS image block (i.e., data point). The moment-based are the mean, variance, skewness, and kurtosis of the distribution of pixel values of an image block (i.e., data point). Finally, spectral clustering can be performed on either set of features to effectively transform a feature vector into a new feature vector, in a lower dimensional space. These 16 wavelet features and 4 moment features are extracted for each data point $(2 \mathrm{~m} \times 2 \mathrm{~m}$ area of seabed).

We perform unsupervised seabed segmentation on a MUSCLE SAS image that spans an area of $56 \mathrm{~m} \times 56 \mathrm{~m}$ of seabed. To allow an assessment of the segmentation results, we manually ground truth this SAS image, shown in Figure 6, into three seabed types (namely, flat, rippled, and rocky seabed). The result of this ground-truthing is also shown in Figure 6. 

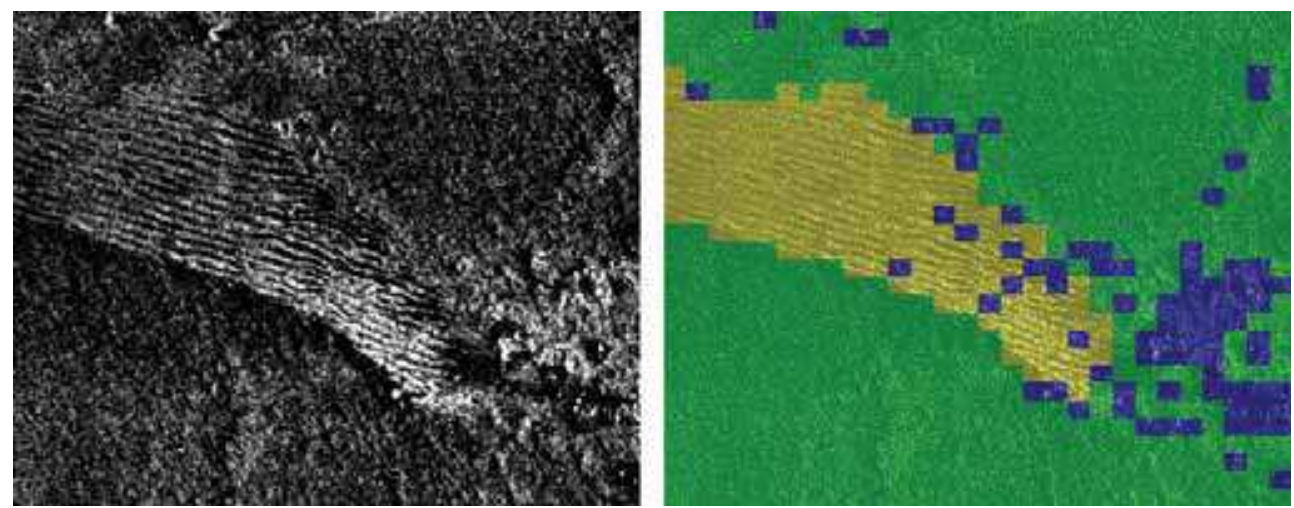

Fig. 6. (a) A SAS image, and (b) its corresponding ground truth (where green, yellow, and blue correspond to flat, rippled, and rocky seabed, respectively).

In the four feature and clustering combinations considered, the segmentation process is completely unsupervised, since it is assumed that no training data is available. The number of clusters to be learned is fixed at $k=4$. Because the $k$-means algorithm is not guaranteed to result in the globally optimal clustering, 100 random cluster-centroid initializations are considered for each case. The clustering for which the distortion - defined as the sum of distances from each point to its assigned cluster centroid - is a minimum is selected as the final clustering (and by extension, the final segmentation).

The results of the unsupervised seabed segmentation on the SAS image shown in Figure 6 are shown in Figure 7. Because the methods are unsupervised, no explicit correspondence between clusters and seabed types exists. However, for purposes of evaluating the segmentation results here, one can easily assign a correspondence between ground-truth seabed types and clusters.

\section{Conclusion}

In this chapter, an overview of different automatic seabed classification approaches has been provided. Although in some cases a completely automated model-based method is possible (when the phenomenon to identify can be modelled mathematically), in most situations some degree of human intervention is required. In the supervised case, initial training is required to drive the system's focus to the classes of interest, whereas in the unsupervised case the operator should ratify the soundness of the class division suggested by the system. It can even be argued that the model-based case also requires human intervention, since the model itself must be created in the first place.

In any case, it has been shown that the three general classification approaches are extremely useful for the automatic processing of data collected in AUV sonar missions, which frequently consist of hundreds or thousands of images that would otherwise require manual processing. This automation allows operators to focus on higher-level tasks, such as mission planning or model design, which could make seabed surveillance operations simpler and more effective. 

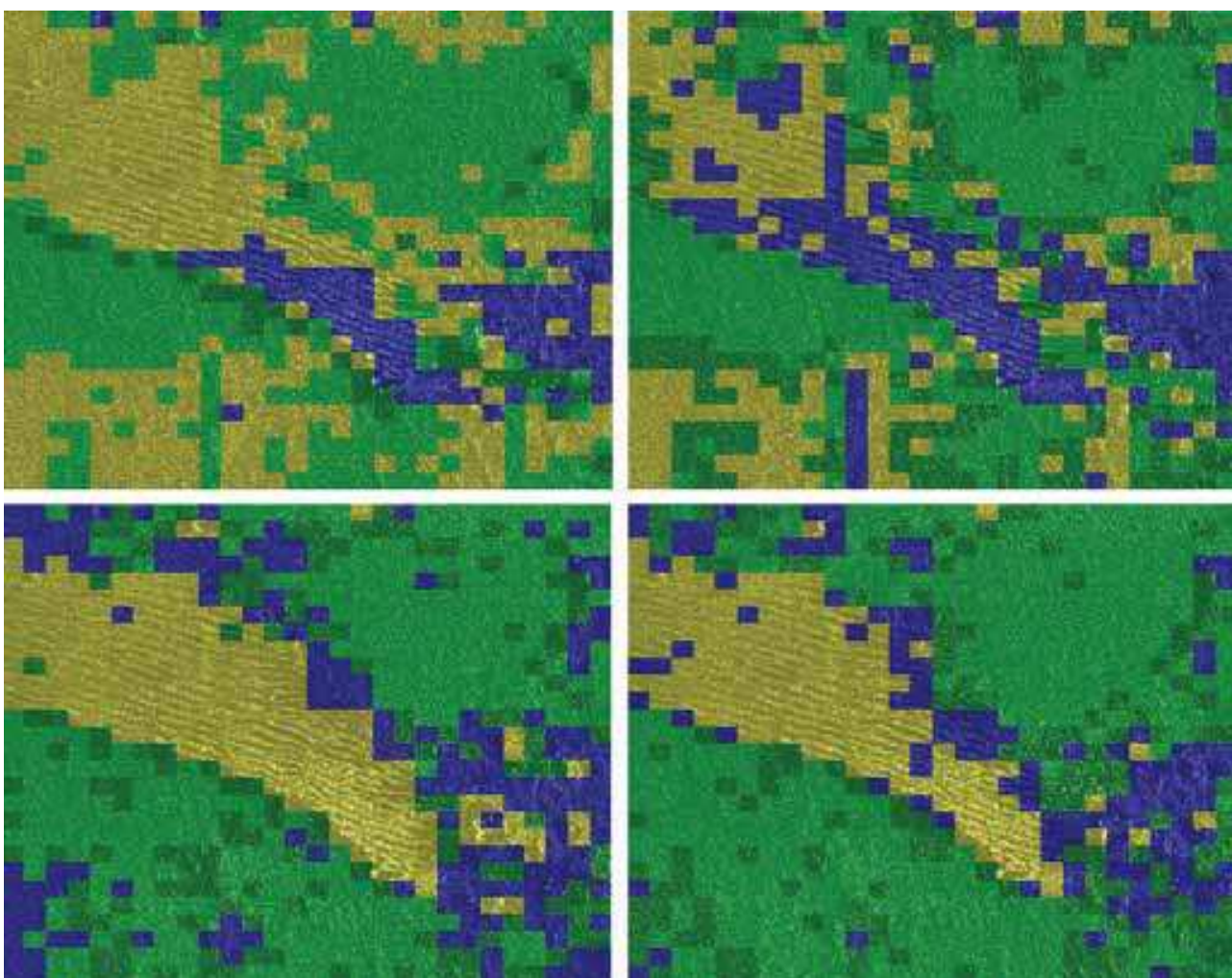

Fig. 7. Results of unsupervised seabed segmentation algorithms. Clockwise from upper left: results from using (a) moment features with $k$-means, (b) moment features with spectral clustering, (c) wavelet features with spectral clustering, and (d) wavelet features with $k$ means.

\section{References}

Berger, J. (1993). Statistical Decision Theory and Bayesian Analysis, Springer. Breiman, L. (1993). Classification and Regression Trees, Chapman \& Hall.

Chang, T., Kuo, C.-C.J. (1993). Texture Analysis and Classification with Tree-structured Wavelet Transform, IEEE Transactions on Image Processing, Vol. 2, No. 4, pp. 429441.

Coiras, E., Myers, V. \& Evans, B. (2007). Reliable Seabed Characterization for MCM Operations, Proceedings of the IEEE/MTS Oceans'07 Conference.

Lewis, J.P. (1995). Fast Template Matching, Vision Interface, p. 120-123.

Liao, X., Xue, Y., and Carin, L. (2005). Logistic Regression with an Auxiliary Data Source, Proceedings of the 22nd International Conference on Machine Learning, pp. 505512.

Mallat, S. (1999). A Wavelet Tour of Signal Processing, Academic Press. 
Meila, M. \& Shi, J. (2000). Learning Segmentation by Random Walks, Advances in Neural Information Processing Systems, pp. 873-879, MIT Press.

$\mathrm{Ng}$, A., Jordan, M., and Weiss, Y. (2001). On Spectral Clustering: Analysis and an Algorithm, Advances in Neural Information Processing Systems, pp. 849-856, MIT Press.

Rasmussen, C. \& Williams, C. (2006). Gaussian Processes for Machine Learning, MIT Press.

Shafer, G. (1976). A Mathematical Theory of Evidence, Princeton University Press.

Shawe-Taylor, J. \& Cristianini, N. (2000). An Introduction to Support Vector Machines: and Other Kernel-based Learning Methods, Cambridge University Press.

Sugiyama, M., Krauledat, M., and Muller, K. (2007). Covariate Shift Adaptation by Importance Weighted Cross Validation. Journal of Machine Learning Research, Vol. 8, pp. 985-1005.

Tipping, M. (2001). Sparse Bayesian Learning and the Relevance Vector Machine. Journal of Machine Learning Research, Vol. 1, pp. 211-244.

Widmer, G. \& Kubat, M. (1996). Learning in the Presence of Concept Drift and Hidden Contexts. Machine Learning, Vol. 23, pp. 69-101.

Zadrozny, B. (2004). Learning and Evaluating Classifiers Under Sample Selection Bias, Proceedings of the 21st International Conference on Machine Learning. 


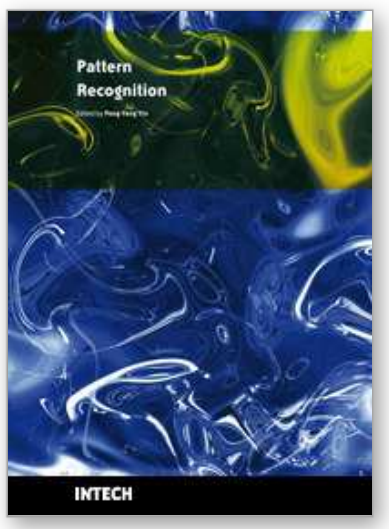

\section{Pattern Recognition}

Edited by Peng-Yeng Yin

ISBN 978-953-307-014-8

Hard cover, 568 pages

Publisher InTech

Published online 01, October, 2009

Published in print edition October, 2009

For more than 40 years, pattern recognition approaches are continuingly improving and have been used in an increasing number of areas with great success. This book discloses recent advances and new ideas in approaches and applications for pattern recognition. The 30 chapters selected in this book cover the major topics in pattern recognition. These chapters propose state-of-the-art approaches and cutting-edge research results. I could not thank enough to the contributions of the authors. This book would not have been possible without their support.

\section{How to reference}

In order to correctly reference this scholarly work, feel free to copy and paste the following:

Enrique Coiras and David Williams (2009). Approaches to Automatic Seabed Classification, Pattern Recognition, Peng-Yeng Yin (Ed.), ISBN: 978-953-307-014-8, InTech, Available from:

http://www.intechopen.com/books/pattern-recognition/approaches-to-automatic-seabed-classification

\section{INTECH}

open science | open minds

\section{InTech Europe}

University Campus STeP Ri Slavka Krautzeka 83/A 51000 Rijeka, Croatia Phone: +385 (51) 770447

Fax: +385 (51) 686166

www.intechopen.com

\section{InTech China}

Unit 405, Office Block, Hotel Equatorial Shanghai No.65, Yan An Road (West), Shanghai, 200040, China 中国上海市延安西路65号上海国际贵都大饭店办公楼405单元

Phone: +86-21-62489820

Fax: $+86-21-62489821$ 
(C) 2009 The Author(s). Licensee IntechOpen. This chapter is distributed under the terms of the Creative Commons Attribution-NonCommercial-ShareAlike-3.0 License, which permits use, distribution and reproduction for non-commercial purposes, provided the original is properly cited and derivative works building on this content are distributed under the same license. 